\title{
Corrigendum: The ATP-P2X Signaling Axis Is an Essential Sentinel for Intracellular Clostridium difficile Pathogen-Induced Inflammasome Activation
}

\author{
Ya-Hui Liu ${ }^{1,2}$, Yung-Chi Chang ${ }^{1}$, Liang-Kuei Chen ${ }^{1}$, Po-An Su ${ }^{3,4}$, Wen-Chien Ko ${ }^{5,6,7}$, \\ Yau-Sheng Tsai ${ }^{8,9}$, Yi-Hsuan Chen ${ }^{1}$, Hsin-Chih Lai ${ }^{10,11,12}$, Cheng-Yeu Wu ${ }^{13,14}$, \\ Yuan-Pin Hung ${ }^{15}$ and Pei-Jane Tsai ${ }^{1,2,16 *}$
}

${ }^{1}$ Department of Medical Laboratory Science and Biotechnology, College of Medicine, National Cheng Kung University, Tainan, Taiwan, ${ }^{2}$ Department of Pathology, National Cheng Kung University Hospital, Tainan, Taiwan, ${ }^{3}$ Division of Infectious Diseases, Chi Mei Medical Center, Tainan, Taiwan, ${ }^{4}$ Department of Pharmacy, Chia Nan University of Pharmacy and Science, Tainan, Taiwan, ${ }^{5}$ Department of Internal Medicine, National Cheng Kung University Hospital, Tainan, Taiwan, ${ }^{6}$ Center for Infection Control, National Cheng Kung University Hospital, Tainan, Taiwan, ${ }^{7}$ Department of Medicine, College of Medicine, National Cheng Kung University, Tainan, Taiwan, ${ }^{8}$ Institute of Clinical Medicine, College of Medicine, National Cheng Kung University, Tainan, Taiwan, ${ }^{9}$ Cardiovascular Research Center, College of Medicine, National Cheng Kung University, Tainan, Taiwan, ${ }^{10}$ Department of Medical Laboratory Science and Biotechnology, Chang Gung University, Taoyaun, Taiwan, ${ }^{11}$ Research Center for Industry of Human Ecology, College of Human Ecology, Chang Gung University of Science and Technology, Taoyaun, Taiwan, ${ }^{12}$ Graduate Institute of Health Industry and Technology, College of Human Ecology, Chang Gung University of Science and Technology, Taoyaun, Taiwan, ${ }^{13}$ Center for Molecular and Clinical Immunology, Chang Gung University, Taoyaun, Taiwan, ${ }^{14}$ Research Center of Bacterial Pathogenesis, Chang Gung University, Taoyaun, Taiwan, ${ }^{15}$ Department of Internal Medicine, Tainan Hospital, Ministry of Health and Welfare, Tainan, Taiwan, ${ }^{16}$ Center of Infectious Disease and Signaling Research, National Cheng Kung University, Tainan, Taiwan

Keywords: Clostridium difficile, inflammasome activation, pyroptosis, ATP-P2X 7 pathway, MyD88

Specialty section: This article was submitted to

Bacteria and Host,

a section of the journal Frontiers in Cellular and Infection Microbiology

Received: 29 June 2019 Accepted: 04 July 2019

Published: 17 July 2019

Citation: Liu Y-H, Chang Y-C, Chen L-K Su P-A, Ko W-C, Tsai Y-S, Chen Y-H, Lai $H-C$, Wu C-Y, Hung Y-P and

Tsai P-J (2019) Corrigendum: The

ATP-P2X 7 Signaling Axis is an

Essential Sentinel for Intracellular Clostridium difficile Pathogen-Induced Inflammasome Activation.

Front. Cell. Infect. Microbiol. 9:260.

doi: 10.3389/fcimb.2019.00260

\section{A Corrigendum on}

The ATP-P2X $\mathrm{X}_{7}$ Signaling Axis Is an Essential Sentinel for Intracellular Clostridium difficile Pathogen-Induced Inflammasome Activation

by Liu, Y.-H., Chang, Y.-C., Chen, L.-K., Su, P.-A., Ko, W.-C., Tsai, Y.-S., et al. (2018). Front. Cell. Infect. Microbiol. 8:84. doi: 10.3389/fcimb.2018.00084

In the published article, there was an error in affiliations 1, 7, 8, and 9. All instances of "Medical College" used in these affiliations should be "College of Medicine."

The authors apologize for this error and state that this does not change the scientific conclusions of the article in any way. The original article has been updated.

Copyright (C) 2019 Liu, Chang, Chen, Su, Ko, Tsai, Chen, Lai, Wu, Hung and Tsai. This is an open-access article distributed under the terms of the Creative Commons Attribution License (CC BY). The use, distribution or reproduction in other forums is permitted, provided the original author(s) and the copyright owner(s) are credited and that the original publication in this journal is cited, in accordance with accepted academic practice. No use, distribution or reproduction is permitted which does not comply with these terms. 